\title{
A Brief History of Music, Computers and Thinking: 1972-2015
}

\author{
Jeanne Bamberger ${ }^{1}$
}

Published online: 29 July 2015

(C) Springer International Publishing 2015

\begin{abstract}
The inaugural issue of the International Journal of Computers and Mathematical Learning (IJCML) appeared in 1996. Now, nearly 20 years later, Digital Experiences in Mathematics Education (DEME) arises like a Phoenix from its ashes. I would like to use the occasion to reflect, on the travels of Logo and specifically MusicLogo beginning with the 1970s when it was first taking shape in the Logo Lab at MIT, through its status as reported in the 1996 IJCML. Then, looking back from here, 20 years later again, the scene looks quite different: MusicLogo has disappeared having morphed into Impromptu, and Impromptu is now available on an iPad. But this journey is just a small instance of related travels that I hope will be pursued by others - for example why did Logo, the original progenitor, disappear as it morphed into Scratch, Netlogo, Boxer and more?
\end{abstract}

Keywords $\operatorname{Logo} \cdot$ MusicLogo $\cdot$ Impromptu $\cdot$ Computer programming $\cdot$ Notations

The inaugural issue of the International Journal of Computers for Mathematical Learning (IJCML) appeared in 1996. Now, nearly 20 years later, Digital Experiences in Mathematics Education (DEME) arises like a Phoenix from its ashes. I would like to use the occasion to reflect on the travels of Logo, and specifically MusicLogo, beginning with the 1970s when it was first taking shape in the Logo Lab at MIT, through its status as reported in the inaugural issue of IJCML (Bamberger 1996). Looking back from here, some 20 years later again, the scene looks quite different: MusicLogo has disappeared having morphed into Impromptu, and Impromptu will soon be available for the iPad.

Electronic supplementary material The online version of this article (doi:10.1007/s40751-015-0003-3) contains supplementary material, which is available to authorized users.

Jeanne Bamberger

jbamb@mit.edu

1 Music and Urban Education, Massachusetts Institute of Technology, Emerita, Cambridge, MA, USA 
But this journey is just a small instance of related travels that I hope will be pursued by others - for example, why did Logo, the original progenitor, disappear as it morphed into Scratch, Netlogo, Boxer and more? ${ }^{1}$

The MusicLogo story began in 1972 when I joined the Logo Lab at MIT. In spite of the changes in technological means that have emerged over all this time, the goals of that first development have remained focused on the same few powerful ideas.

- To design educational environments that will help students value their powerful know-how, make it explicit, and build on what they already know how to do.

- To give students, especially those who do not yet play an instrument, the possibility actually to make music.

The ambience in the Logo Lab community in 1972 offered me a kind of paradigm for what a more challenging and more effective learning environment could be. It was intellectually luxurious, but like my own inquiries it seemed to be a luxury of necessity. If we seriously wanted to influence the level of engagement of our students, even the place, the value of learning, then this new computer-oriented environment suggested the necessity of considering what is possible not just what is practical. It meant probing assumptions, facing ambiguities, asking hard questions - all things we actually do in our own practice as researchers, but too frequently leave outside the door when we enter the classroom. The remarks that follow trace my way along the path in search of computer environments that would meet these demands.

The reader should bear in mind that in the early 1970s when we began work with Logo and music, the computer was still a very new and rather strange, even somewhat frightening machine. We were working with the DEC PDP-10 computer and somewhat later the PDP-11. These were huge machines, totally filling a room the size of a concert hall big enough to hold 100 people. Moreover, the notion of a 'personal' computer was not even imagined. So, as I recount events in the 1970s and 1980s, the descriptions of students at work and the computers facilitating it may give readers a slightly creepy, not quite believable, feeling of time warp. Just as one example, here is a description by a student working with Logo in the 1970s:

The gadgetry is quite simple for the user: He finds himself at a typewriter that is connected to a computer by phone line, the computer itself is somewhere else.

(Bamberger 2013, Chapter 11, p. 176)

Given this image, you can imagine my disbelief when, a few years later, a student came into my office to tell me he was going to the MIT bookstore (The Coop) to buy a computer. And he did.

\footnotetext{
${ }^{1}$ In an opening statement on an un-numbered page in the then-new journal IJCML, the Editors commented on the future both of the journal and of computers. The vision of the journal now seems at once strangely arcane and, in ways most probably not intended or anticipated, prescient: We believe that computers, and the ideas and cultures engendered by them, will have a critical role in the far-reaching changes that will come. [...] Furthermore, we expect to see these changes having major impacts on the organization and structure of schooling, on approaches to assessment and on professional development.
} 


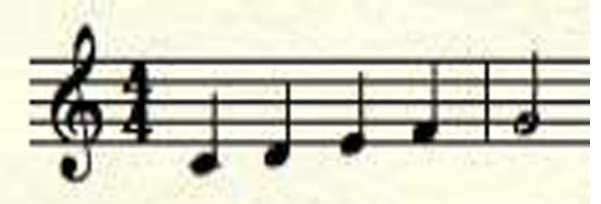

Fig. 1 The sounding result of the PLAY procedure

\section{Musiclogo}

The first incarnation of MusicLogo included a full Logo language with the addition of one music primitive, NOTE. (Terry Winograd, then a graduate student in the AI Lab, wrote NOTE one night.) NOTE took two inputs, :P :D, for Pitch and Duration. From NOTE we made PLAY, which took two lists, a Pitch list and a Duration list, thus: PLAY [ $\left.\begin{array}{lllll}1 & 2 & 3 & 4 & 5\end{array}\right]\left[\begin{array}{lllll}6 & 6 & 6 & 6 & 12\end{array}\right]$ which played: (Fig. 1).

\section{Basic Musiclogo Procedures}

The following are very simple, straightforward MusicLogo procedures. However, they contain a critical aspect of the Logo approach to learning -discovering and abstracting common structures amidst quite different media or situations. Consider the procedure, TO COUNTDOWN, which simply produces a print-out of numerals "going down" from some :START number to some :DONE number.

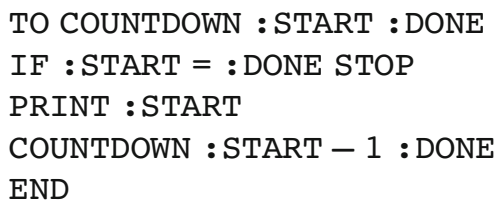

The output or result of running the procedure with inputs of 12 for :START and 1 for :DONE is:

COUNTDOWN 121

12

11

10

9

8

7

6

5

4

3

\footnotetext{
${ }^{2}$ LOGO procedures begin with the infinitive form of the verb, TO, in order to illustrate that the procedure is going to "teach" the computer how TO do some action - here, play or countdown. Inputs or variables are preceded by a colon (called "dots"), as in :P or :START.
} 


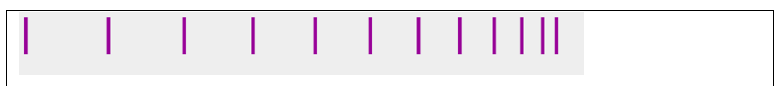

Fig. 2 A spatial analog of the BOOM sound after running the procedure FASTER (Supplementary Audio 1)

1

The same procedure, now called TO FASTER, substitutes only the command $\mathrm{BOOM}$ for PRINT. It produces a BOOM drum sound that gets faster and fasteri.e. each BOOM is one duration shorter than the previous. (Think of it as a ping-pong ball dropped on the floor and continuing to bounce on by itself.)

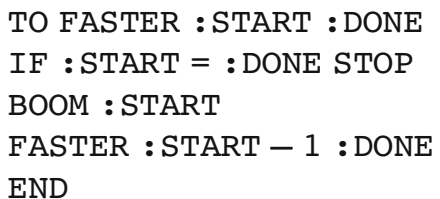

\section{FASTER 121}

(Fig. 2)

The next procedure is again exactly the same except that PLAY is substituted for BOOM. The result is a series of descending pitches in which each pitch is one step down (lower) from the previous pitch. The graphics below show the descending pitches.

TO DOWNPITCH :START : DONE

IF $:$ START $=:$ DONE STOP

PLAY : START - 1 : DONE

DOWNPITCH :START - 1

END

(Fig. 3)

These procedures are descriptions-of-actions that make what they describe. Each procedure "teaches" the computer to use different media to create different effects, but each is brought into action in the same way. Thus, in reflecting on this group of procedures, a general shared strategy emerges - how to "go down" in numbers, time and pitch. As such, the group of procedures reveals and shares conceptual underpinnings.

\section{Learning With Musiclogo}

The story of MusicLogo as it developed, how it was used and what the students were learning is best told by the MIT students themselves. The quotations given below are from student work on a final project from an MIT Music Department

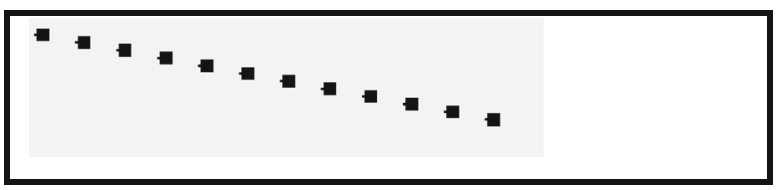

Fig. 3 Pitch descending (Supplementary Audio 2) 
course called Developing Musical Structures. Many of these students were Computer Science majors, so they were able to put the procedural power of Logo to work in an advanced and vital way. As I will show, their work illustrates paradoxically both the power of Logo and also aspects that led to its demise. The task for the final project is well described by a student I will call Chris, who began his final paper as follows:

This is a report on the procedural analysis of the first movement of Vivaldi's Concerto, Summer. The analysis consisted of two parts:

1. A graphical analysis of the piece (Fig. 4).

2. A procedural implementation of that graphical analysis using the Logo music language.

Chris listened to the Vivaldi movement closely and attentively over a period of several days. Later, in reflecting on his very detailed graphical analysis, he remarked about certain graphical figures:

I noticed that they all had something in common which I could express with the following abstraction:

\section{SERIES $: V: N S: I N C: K: U N I T: H O L D$}

which produces a series of notes where:

- $\quad: V$ is the voice that will take the notes;

- $\quad$ :NS is the starting note of the series;

- $\quad$ :INC is the increment that is added to the notes at each step (i.e., would be 1 for a scale);

- $: K$ is the number of notes to be produced;

- : $: U N I T$ is the duration of all the notes;

- :HOLD is the duration of the last note.

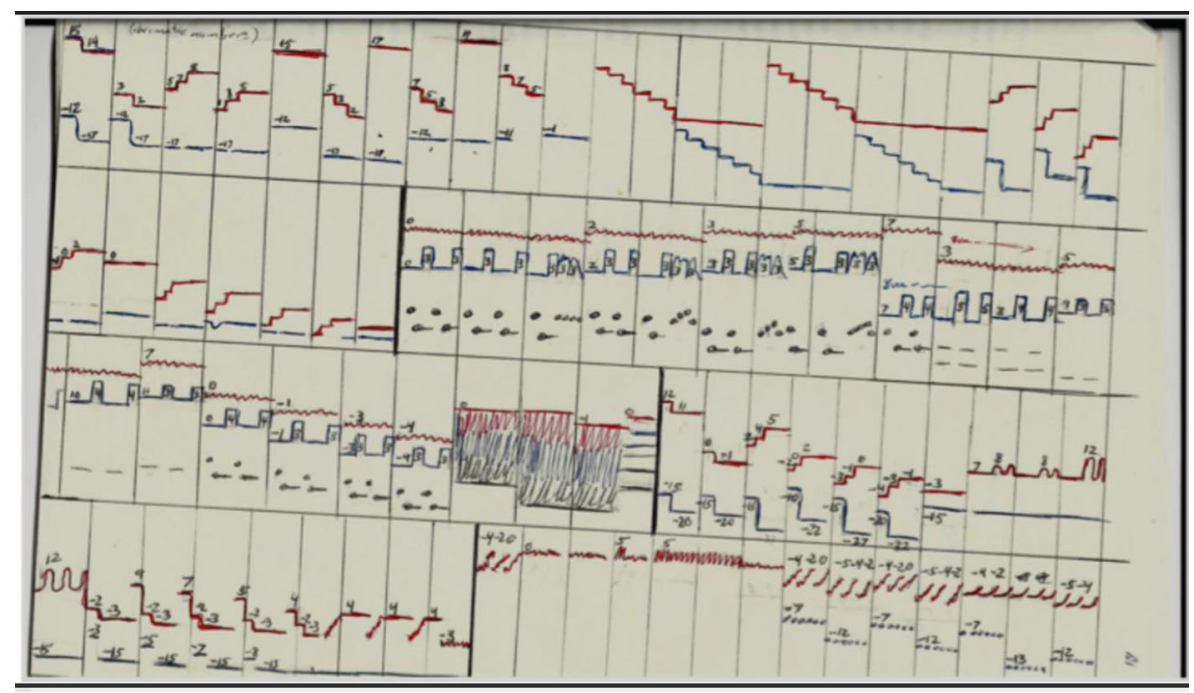

Fig. 4 Graphic analysis of Vivaldi's Summer, 1st movement (Supplementary Audio 3) 
The structure that Chris discovered, which he called "SERIES," describes and also produces what in conventional music terminology is called a "sequence." A sequence is roughly described as a melodic pattern that, as an entity, moves up or down some number of times along a scale or some other small pitch interval. This interval as input to his procedure Chris called :INC. His inputs or variables, noted by a colon or "dots," describe and, as used in the procedure, determine (play) the constituents that form the general structure, sequence. Chris's SERIES procedure (moving up) was as follows:

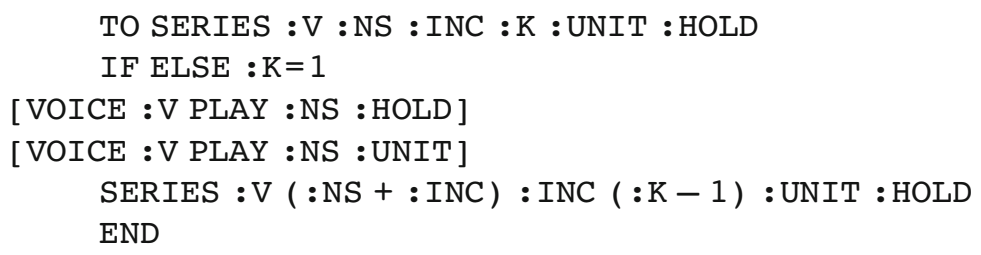

The procedure, SERIES, as a generalized sequence procedure, turns out to be the most characteristic structural element of Vivaldi's works. The class as a group called the procedures they wrote, "Vivaldi's intuitive tool kit." Chris put it this way:

The fact that one can implement so many things with the SERIES abstraction is revealing about Vivaldi's style in this movement [...] First, it emphasizes the importance of sequences of notes going up or down the scale (or some other factor) whether a little or a lot [...] This idea of a series which is held at the end pervades the movement, and thus the SERIES abstraction (Fig. 5).

In a hand-written addendum, Chris made some further remarks that help to inform the power of MusicLogo but, as suggested earlier, perhaps also implicitly led to its disappearance:

The thing that I did not mention is that I am a computer science major here at MIT. The effect of this lies in the effect that my consequent background in the field had on my procedural implementation. In computer science we must forever be on the lookout for abstractions and generalizations. It is this training of mine that enabled me to detect the "series" abstraction for this movement. It is not something I heard on the tape.

It is clear from this small bit of evidence, that thinking procedurally, making a procedural description in the static, discrete symbolic language of MusicLogo, has the remarkable ability to shape the perception of the music as it is organizing and evolving through time ("It is not something I heard on the tape").
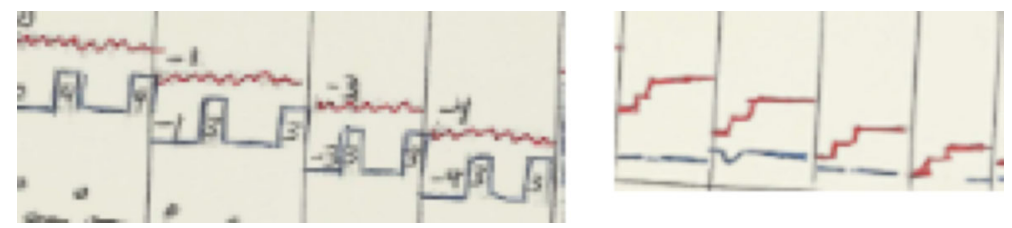

Fig. 5 Sequences (Supplementary Audio 4) 
Another student, Terry Dash (not a computer science major), described the learning process this way:

We learned, finally and perhaps with difficulty, that our pursuit of music was both a pursuit of music and a pursuit of our thinking. As we changed, the music changed [...] There was no framework to what we learned; no framework that held all the pieces together; no pretty function that defined the system. Rather, there was only a process of learning, of discovering possible structures and viewpoints; a multiplicity of frameworks in which to work; a set of functions in which all variables were dependent.

\section{MusicLogo Morphing into Impromptu}

This makes a nice segue into the development of Impromptu. Looking back to Impromptu as I wrote about it in that first issue of IJCML, I see three primary reasons why MusicLogo disappeared and why it morphed into Impromptu.

* First (implicit in what both Chris and Terry wrote), for Chris, his background as a Computer Science major was, by his own words, a critical factor in working with MusicLogo - he had learned "forever to be on the lookout for abstractions and generalizations." And Terry commented that: “... perhaps with difficulty our pursuit of music was both a pursuit of music and a pursuit of our own thinking." Unfortunately, a search for "abstractions and generalizations" or a "pursuit of (one's) own thinking" are rarely found as primary goals of instruction in everyday school classrooms today music or otherwise. In short, our design of MusicLogo as an environment for "teaching children thinking" was simply not practical. ${ }^{3}$

* Second, with respect to learning, MusicLogo procedures are initially silent symbolic descriptions with only the potential for becoming organized sound. Thus, in order to hear the results of their thinking (or inner hearing) in sound, students had to learn to "instruct" the computer with symbolic descriptions in a language, Logo, that the computer could "understand." This too required "thinking abstractly."

* Third, by the 1990s, technology had rapidly developed to include the capacity for students to work directly and immediately with sound. With these technological advances, no descriptions were needed: students could start with listening to preprogrammed music by simply clicking on graphical objects on the computer screen and, along with "drag and drop," easily manipulate the sounding music that the screen objects represented and produced. In short, there were immediate, ready-at-hand "products."

Given these non-practical issues of MusicLogo, together with the advances in technology, we needed to ask, what could be gained and what might be lost if MusicLogo morphed into something more useable? But there was a cautionary note. There were music programs readily available such as Garage Band, Reason, Guitar Hero and others that could make many musical decisions for you: in this context, thinking tends to slip away with the ease of the app.

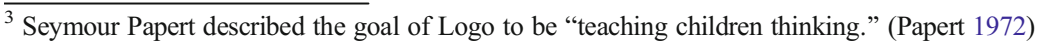




\section{Developing Impromptu}

While it was in 1972 that the student found himself "at a typewriter that is connected to a computer by phone line," our goals in 1996, despite the dramatic technological developments, remained much the same. As in 1972, our aim was still '... to help students value their powerful know-how, make it explicit, and build on what they already know how to do; to help students confront real and sometimes problematic musical situations of their own and others' making; to work to erase that sometimes painful distinction between learning about music and doing it; and through reflection, to come to understand and participate more fully in the complexity of complex music.'

The development of Impromptu, as it emerged in the early 1990s, was influenced by two primary factors.

1. My earlier studies (see Bamberger 2013, Part 1) clearly demonstrated that the kinds of elements and relations novices attend to in making sense of music, as it unfolds in real time, are highly aggregated, structurally meaningful entities such as motives, figures and phrases. These are the 'units of perception' - the elements that novices have ready access to, their focus of attention. Similar to talking, in conversation we do not pay attention to syllables but rather group them into meaningful words, the words taking on particular meaning as they group into phrases and sentences. Just so, in listening to melodies, we do not hear individual notes, but rather the inner motion of groupings of notes moving to their goals as they coalesce into motives or small "figures."

2. Through listening to music from our own culture, we have become most responsive to structural functions such as stability and instability - whether a phrase sounds ended or is still going on; even, given a context, whether a note sounds at rest or not.

With these findings in mind, and benefitting from the advances in computer technology, I was able to do what we had never been able to do before, namely to program these structurally meaningful groupings and represent them on the screen as graphic icons. When clicked, the icons play melodic figures that are our intuitive units of perception. Moreover, with drag and drop, users could manipulate these structural units arranging and rearranging them in the "Playroom" to reconstruct tunes and to compose new ones. 4 Students could work directly with the icons as units of perception, units of description, and units of work. We called them "tuneblocks." (Bamberger 1996) (Fig. 6).

Typical of projects given to students using tuneblocks was the following.

A First Task:

Using a given set of pre-programmed tuneblocks, select and drag blocks into the Playroom, putting them in order so as to reconstruct the whole given tune.

With this task, a given tune and its set of tuneblocks as the working materials (chosen from both familiar and unfamiliar melodies), the nature of learning as compared with MusicLogo was dramatically transformed. Procedural thinking, with its text-driven abstractions and generalizations, was replaced with what became a live "conversation" with sounding materials of music. By "conversation," I mean the silent conversations we have with hands-on materials as we are building, fixing, or inventing objects that work. Working with

\footnotetext{
${ }^{4}$ In addition we designed graphics that showed the contents of these perceptual units as "pitch-time shapes" see Fig. 6.
} 


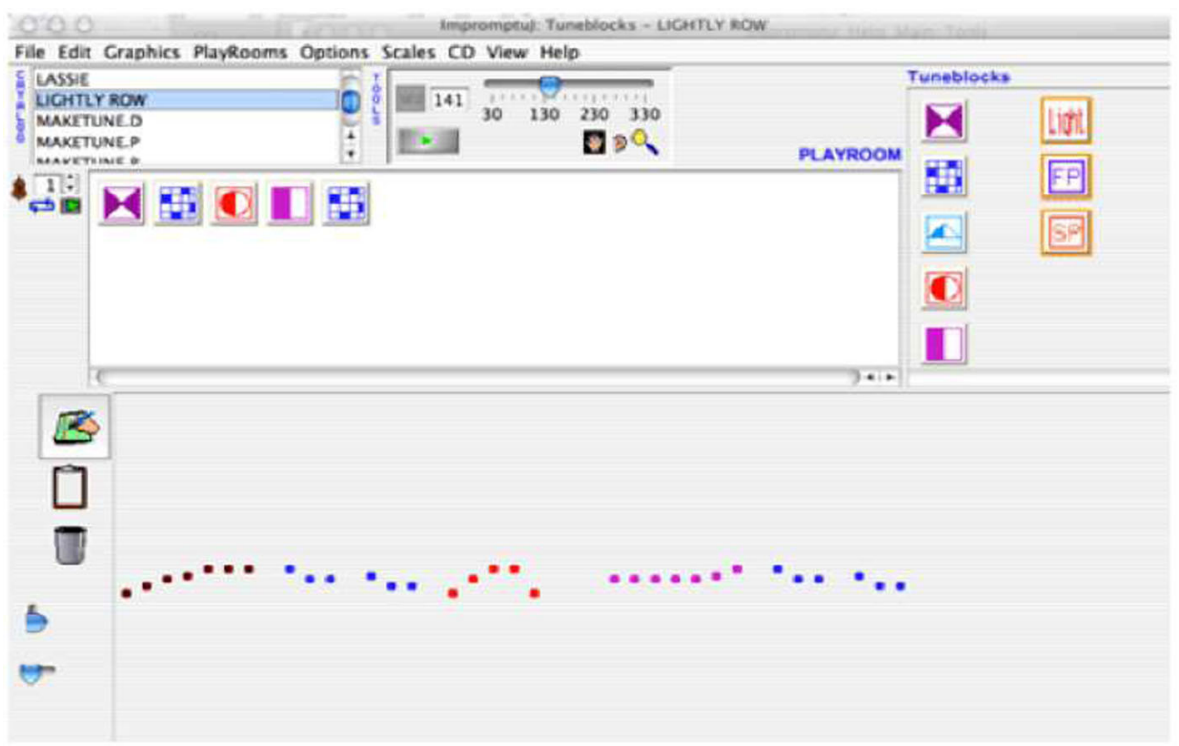

Fig. 6 The tuneblocks window

tuneblocks, like working with hands-on materials, we learn as we listen to these materials, arranging and rearranging them, watching them take shape even as we shape them. The blocks "talk back" to us remaking our ideas of what is possible. The back-talk leads to new actions on our musical objects in a spiral of inner and outer activity: our inner intentions are reflected back by the results of our actions, leading to new outer actions and often to changing of our intentions. It is a kind of "re-search" - one that is as familiar to the scientist designing a theory as to the painter or composer designing an artifact. Arnold Schoenberg (1975), juxtaposing the "technique of musical composition" with "carpentry," put it this way:

For if the carpenter knows how to join pieces of wood securely, this knowledge is based no less on fruitful observation and experience than is the knowledge of the [composer] who understands how to join chords effectively. And if the carpenter knows which types of wood are required by a particular job and selects accordingly, he is thus taking natural relationships and materials in to account, just as does the [composer] when, appraising the possibilities of themes, he recognizes how long a piece may be. (p. 385)

What was gained and what was lost as MusicLogo morphed into Impromptu? The process of learning was, itself, almost reversed. Chris and Terry, working with MusicLogo, began with close listening to a complex piece of music. Through analysis expressed first in graphics, they derived, generalized, described procedurally and made Vivaldi's shared structures into sound - Vivaldi's intuitive tool kit. In contrast, students began work with Impromptu as action experiments or what we called constructive analysis (in contrast to destructive analysis). ${ }^{5}$ Reconstructing familiar tunes and later composing their own with

\footnotetext{
${ }^{5}$ Impromptu includes an edit function that allows users to open up blocks to look at their "contents" - i.e., the actual pitches and their durations.
} 
tuneblocks as the units of perception and of work, they made melodies that "worked." Critically important, they watched themselves at work, reflecting on their decisions and asking themselves why. As one student put it at the end of her paper:

A big question in my mind: what makes a certain sequence of notes, blocks sound like an ending? We see that most people agree on what things have ending sounds, so what makes it that way?

Her question followed us throughout the rest of the semester.

\section{Learning with Impromptu}

As students worked with Impromptu making tunes and only then reflecting on their results, they were, indeed, making explicit their intuitive knowledge-in-action and going beyond it. For example, unlike the MusicLogo students who began with the analysis of complex compositions, the Impromptu students found common, shared structures in their reconstruction of the simplest tunes and, in making their own, only discovering them again as generative means in the more complex compositions that we studied later.

As further evidence, notice again in the figures below, the sequential shaped structures that Chris found in his graphic analysis of Vivaldi; then notice that these sequential shapes are also reflected in the graphics for certain given Impromptu tuneblocks. However, there is a big difference in the process of their generation, recognition and use. MusicLogo students abstracted out the sequence as a common structure in complex pieces, later using the power of the computer as a tool to construct a working procedure for the generalized sequence structure. Impromptu students used the power of the computer as a sounding board to listen to pre-programmed structural entities, using them as elements to build up tunes. It was in the process of building-up and reflecting on their emerging tunes that they discovered the sequence as a common structure. Impromptu students make music happen, but not by deriving the formal, generalizable, procedural descriptions that MusicLogo students made of these shared structures (Fig. 7).
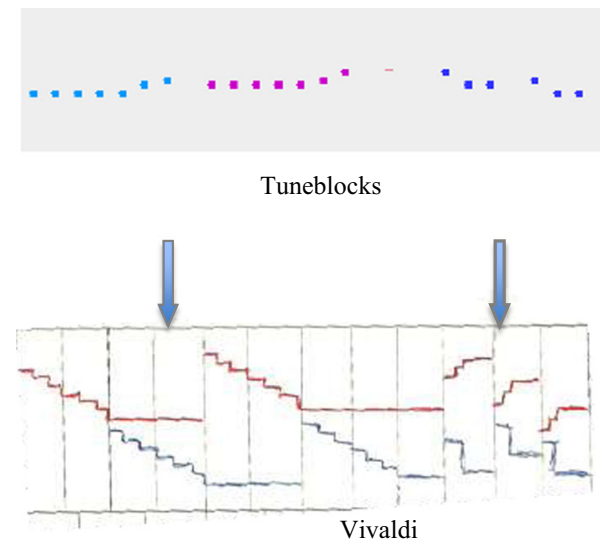

Fig. 7 Sequences (Supplementary Audio 5) 
Was the goal, then, to use the computer as a compositional means or to develop abstract, generalizable principles, or could it be both? In answer, I again quote the work of a student, this time one who was using Impromptu in a class called "Music Cognition." In that class, students worked with a given a set of tuneblocks using them as the materials for composing a song. The students were able to edit the pitches and durations of blocks by simply opening a block in the editor to see and to work with its contents. Through experimenting, listening back and reflecting on the results, students could change pitches and durations to whatever worked for them. But it was critically important that the students were asked to keep a log of their decisions, accounting for them as they went along in an effort to make their own intuitive "mental organizers" explicit.

\section{An Example of Student Work}

Jon started with a set of "strange blocks" called Portals (Supplementary Audio 6).
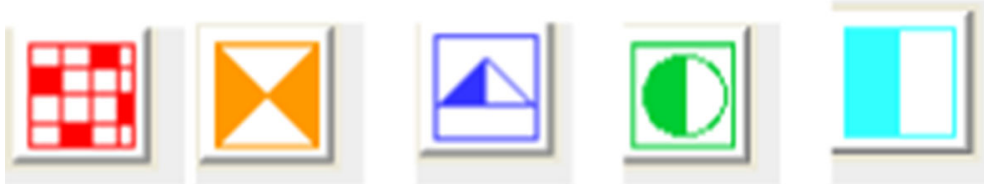

He edited the blocks to create sequences around which he organized his piece. On the Impromptu screen, his edited blocks showed up as groups of likenamed and like-colored blocks, each group forming a sequence. The sequences can also be clearly seen in the spatial analog graphics of his melody shown below (Fig. 8)

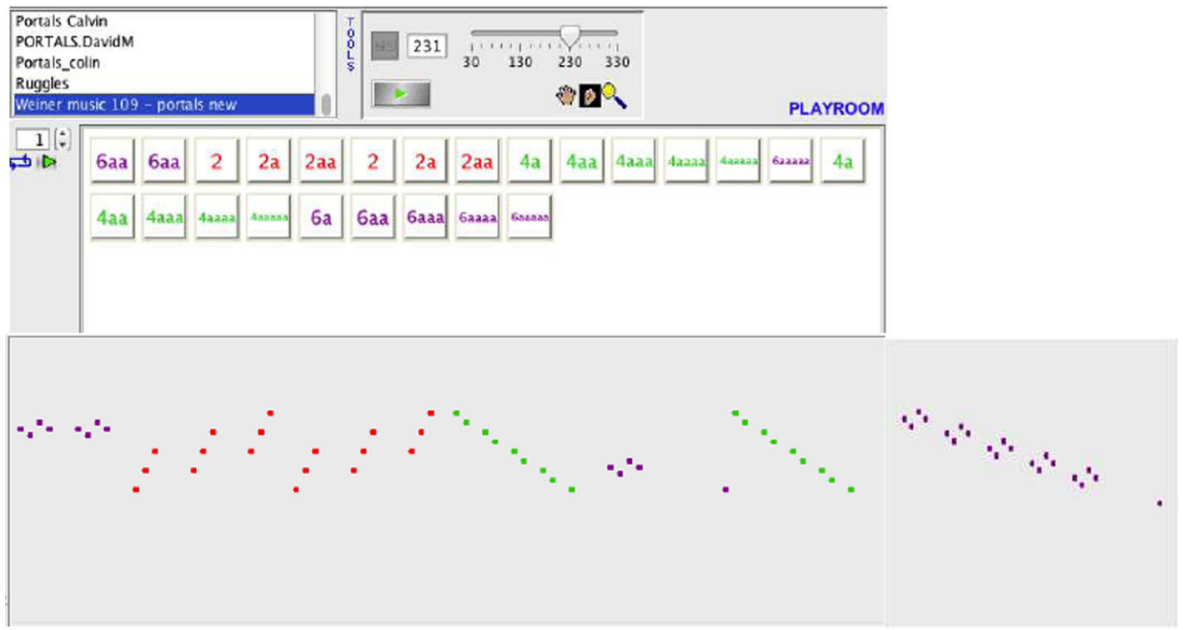

Fig. 8 Jon's sequential composition (Supplementary Audio 7) 
In his reflective paper written from the log he kept during the composition process, Jon made the following comments:

One of the things that we've talked about that can be seen in my melody are sequences, which are all over the place, and really make the foundation of my piece, and repetition. Also of note is the mix of fast notes and pitch space. Most of my sections covered very similar pitch distances, but did so in wildly varying times and manners. That, I think, is what makes this piece so interesting to me. I cover very similar pitch spaces, but manage to change how I do it by varying the intervals and rhythms so that each time up or down is a little bit different.

In a more overall look at the piece, it is actually fairly simple in its construction. The $\mathrm{A}$ and $\mathrm{B}$ sections are simple repetition, and then the $\mathrm{C}$ and $\mathrm{C}$ ' sections just make up an antecendent-consquent pairing. Additionally, the internal structure is mostly sequences and repetition of notes within those series, as can be seen clearly in the e section with the sequence of 4 notes descending, as well as in the b section with the groups of 3 ascending (Fig. 8).

To close, I had a lot of fun working with the blocks in Portals. The funky nature of the blocks in Portals really allowed me to be more creative than I was with more standard blocks like those in other sets, and I feel that allowed my creativity and imagination to bloom and create the unbalanced, balanced, dissonant, and consonant piece that I present.

Notice that Jon referred to a range of different compositional means as he commented on his process and on his composition. Particularly interesting is the attention to his use of "pitch space" which he used both to create coherence (keeping it the same) and, within it, variation. Further, in reflecting on his work, he analyzed and described its fundamental organization on both the detailed and larger design levels. Finally, he concluded with his feelings of satisfaction with the process of composing, as well as with the final product.

While Jon, unlike Chris, worked mostly by experimenting with musical materials, can we say that he has abstracted and generalized? Perhaps, but clearly with different intent and different results when compared with the purposes and satisfactions of Chris and Terry. Recall Chris's comment, "It is this training of mine that enabled me to detect the "series" abstraction for this movement. It is not something I heard on the tape." And Terry: "We learned, finally and perhaps with difficulty, that our pursuit of music was both a pursuit of music and a pursuit of our thinking. As we changed, the music changed." And once more returning to Jon: "I feel [the funky nature of the blocks in Portals] allowed my creativity and imagination to bloom and create the unbalanced, balanced, dissonant, and consonant piece that I present."

So what has been gained with the introduction of Impromptu? Most of all, a working environment where students are actually making music. They are actively using their "sensory/mental organizers" to listen, manipulate, organize and re-organize the going- 
on of sound and time. And they are learning by reflecting on the objects and the process through which their newly coherent objects came into being.

To recapitulate my earlier intentions, Jon made quite clear that the blocks did "talk back," remaking his ideas of what was possible. Moreover, the back-talk led to new actions: inner intentions led to new outer actions changing Jon's intentions in a kind of "re-search" that became his developing sequences. As I had imagined, Impromptu became a working environment mediated by symbolic representations and tools designed to engage students' everyday know-how, helping to make it explicit, while building on their already powerful knowledge-in-action.

\section{Coda}

To come full circle, I ask again, why, did we give up MusicLogo, allowing it to rest quietly in the past? Having traced the path of the journey, a new view emerges: perhaps MusicLogo only seems to be quiet there. Perhaps MusicLogo was and maybe still is functioning as a necessary nurturing ground for Impromptu. Rethinking the past, Impromptu was probably only possible through the "abstractions and generalizations" that emerged from living and learning with students during those early years in the Logo Lab.

For example, MusicLogo taught me to value computers as a playground for thinking, but it also prodded me to build a workshop for making things that would become new objects of reflection. Watching students such as Chris and Terry working with MusicLogo taught me to value the elegance of musical abstractions. But at the same time it made me sad to see sounding time stopped by static symbols and a unique situation become a type, an instance of a class. The students' very insights prodded me to seek alternative means, particularly for confronting the necessary transformations for moving between static generalizations and organized sound disappearing through time. The question became:

What is the nature of transformations such that elegant but static symbolic abstractions may become coherent sounding musical structures even as they disappear in time?

Hasty (1997) puts it this way:

how shall we account for those attributes of [music] that point to the particularity and spontaneity of aesthetic experience as it is happening [...] To the extent we find it comprehensible, music is organized; but this is an organization that is communicated in process and cannot be captured or held fast. (p. 4)

Looking back once more to 1996, and the beginnings of IJCML, I see this journey as revealing a generative paradox rumbling in the background. The high, abstract generalizations of Logo and MusicLogo guided the need, and eventually the design, of a practical music workshop. Impromptu, in response to that need, made it possible for musically innocent students to generate and to reflect upon down-to-earth musical objects whose structures brought alive the abstractions from which they had evolved. Learning became reflective music-making, silently benefitting from the abstract 
thinking of Chris and Terry. They and all the others helped to make MusicLogo a quiet progenitor for today's students.

It remains, now, for others who participated in the grand history of IJCML to look back and reflect on their personal Logo journeys. Hopefully, my path in the music domain will relate to and perhaps intrigue others to follow along. I look forward to more stories. ${ }^{6}$

\section{References}

Bamberger, J. (1996). Turning music theory on its ear. International Journal of Computers for Mathematical Learning, 1(1), 33-55.

Bamberger, J. (2013). Discovering the musical mind. Oxford: Oxford University Press.

Hasty, C. (1997). Meter as rhythm. New York: Oxford University Press.

Papert, S. (1972). Teaching children thinking. Programmed Learning and Educational Technology, 9(5), 245255.

Schoenberg, A. (1975). Style and idea. Berkeley: University of California Press.

\footnotetext{
${ }^{6}$ History repeats: On February 2nd, 2015, in a new video from the Lighthouse Community Charter School titled " 5 th Grade Turtle Art," I read the following: "In 'Turtle Art,' students enter basic commands (such as "forward," and "left") to move a turtle around a screen. The turtle has ink on her tail and, wherever she moves, she draws a line." 